\title{
Introduction to Ultra-Wideband Antennas
}

\author{
Patan Imran Khan \\ Dept. of ECE, Bheema Institute of Technology and Science, Adoni-518301,, Kurnool, A.P. \\ *Correspondence: imrankhan.bitsadoni@gmail.com
}

This paper presents an introduction to ultra-wideband (UWB) antennas, including a summary of key UWB antenna concepts, as well as system and network considerations, and fundamental limits for UWB antennas.

Keywords: UWB antenna, log periodic antenna, tapered horn antenna (top), EIRP.

\section{Introduction}

An antenna is a transducer that converts guided electromagnetic energy in a transmission line to radiated electromagnetic energy in free space. Antennas may also be viewed as an impedance transformer, coupling between an input or line impedance, and the impedance of free space.

The imminent widespread commercial deployment of ultrawideband (UWB) systems has sparked renewed interest in the subject of ultra- wideband antennas. The power levels authorized by the FCC mean that every dB counts in a UWB system - as much or perhaps even more so than in a standard narrowband system. Thus, an effective UWB antenna is a critical part of an overall UWB system design.

UWB antennas have been in active commercial use for decades. In a sense, even the venerable AM broadcast band antenna is "UWB" since it covers a band from $535-1705 \mathrm{kHz}$ for a fractional bandwidth in excess of $100 \%$. Because a high quality broadcast AM antenna is really a tuned antenna designed to pick up an individual narrowband $(10 \mathrm{kHz})$ channel, the effective fractional bandwidth is really only 0.6$1.9 \%$ and only one channel can be received at a time.

This is a particularly stark example, but it highlights the difficulty with traditional UWB antennas: they are typically "multi-narrowband" antennas instead of antennas optimized to receive a single coherent signal across their entire operating bandwidth. Some modulation schemes are more tolerant of antenna variations than others. For instance, a multi-band or OFDM approach may be less vulnerable to dispersion or other variations across an antenna's operational band. Nevertheless, a UWB system requires an antenna capable of receiving on all frequencies at the same time. Thus, antenna behavior and performance must be consistent and predictable across the entire band. Ideally, pattern and matching should be stable across the entire band.

In addition, a UWB antenna is preferentially non-dispersive, having a fixed phase center. If waveform dispersion occurs in a predictable fashion it may be possible to compensate for it, but in general it is desirable to radiate similar waveforms in all directions. A log-periodic antenna is an example of a dispersive antenna. Larger scale components radiate low frequency components while smaller scale components radiate high frequency components. The result is a chirp-like, dispersive waveform. Worse, the waveform will vary at different azimuthal angles around the antenna. Again, a multiband or OFDM approach may be more tolerant of dispersive antennas.

By contrast, a small element antenna, like a planar elliptical dipole tends to radiate a more compact, non-dispersive waveform, similar to a "Gaussian W." This behavior is illustrated in Figure 1. Since a small element antenna not only tends to be non-dispersive, but also more compact, small element antennas are preferred in many applications.
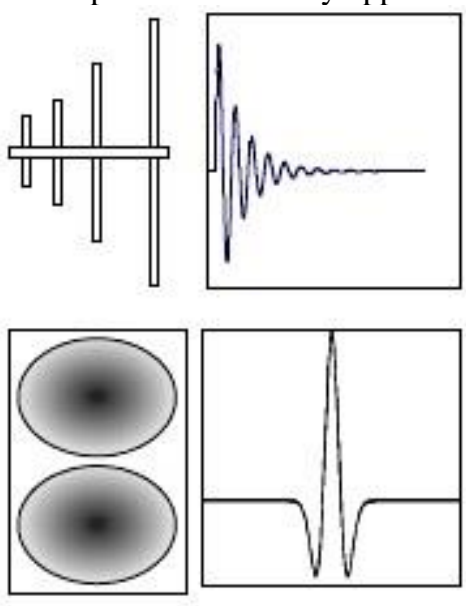

Figure 1: A log periodic antenna (upper left) has a dispersive waveform (upper right), while an elliptical dipole (lower left) has a non-dispersive waveform (lower right). 
The aim of this paper is to provide an overview of and introduction to UWB antennas. This paper will explain key UWB antenna concepts, discuss system and network considerations for UWB antennas, and present fundamental physical limits to UWB antenna performance.

\section{UWB Antenna Concepts:}

A wide variety of antennas are suitable for use in ultrawideband applications. Some of these are described elsewhere in a historical survey. UWB antennas may be classified as directional or non -directional. They may further be classified as either electric or magnetic antennas. These classifications as well as the various types of UWB antennas will be considered in turn.

\section{2a. Directionality of Antennas:}

High gain or directional antennas concentrate energy into a narrower solid angle than an omni-directional antenna. An isotropic antenna has a gain of $0 \mathrm{dBi}$ by definition (such an antenna is not physically realizable, at least not in terms of instantaneous pattern). In fact, "dBi" means $\mathrm{dB}$ relative to an ideal isotropic antenna. A typical dipole antenna has a gain of about $2.2 \mathrm{dBi}$. High gain horn or reflector antennas may have gains of $+10 \mathrm{dBi},+20 \mathrm{dBi}$, or even more (see Figure 2). Antenna efficiency is included in the definition of antenna gain, so a $50 \%$ efficient $(-3 \mathrm{~dB})$ dipole will have a gain of about $-1.8 \mathrm{dBi}$.

A directional antenna will have high gain, a narrow field of view, and will be relatively large in size. An omni-directional antenna has relatively low gain, a wide field of view and will tend to be relatively small. The fundamental trade-offs with directional antennas are shown in Table 1:

\begin{tabular}{|l|l|l|}
\hline Parameter & Directional & $\begin{array}{l}\text { Omni- } \\
\text { Directional }\end{array}$ \\
\hline Gain & High & Low \\
\hline Field of View & Narrow & Wide \\
\hline Antenna Size & Large & Small \\
\hline
\end{tabular}

Table 1: Trade-offs between directional and omni antennas.
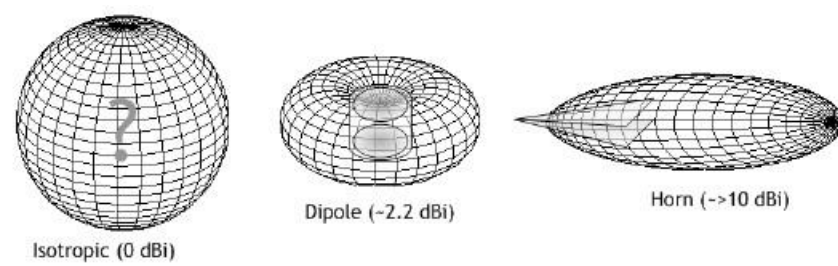

Figure 2: An isotropic antenna (left) has a gain of $0 \mathrm{dBi}$ by definition. A small dipole antenna (center) typically has a gain of about $2.2 \mathrm{dBi}$, and a horn antenna (right) may have a gain of $10 \mathrm{dBi}$ or more.

Note that regulatory constraints require transmit power to be decreased when using a high gain directional transmit antenna so as to meet the same peak radiated emission limit. Thus, a high gain transmit antenna does not add directly to the link budget except in so far as it might reduce emissions in undesired directions. This can potentially reduce clutter and enhance overall system performance or capacity. Of course, a high gain receive antenna adds directly to link performance and is always desirable where the relatively larger size and narrower field of view can be tolerated. These trade-offs are illustrated in Figure 3. Implications of antenna directivity and gain for overall system performance will be discussed in Section 3B.

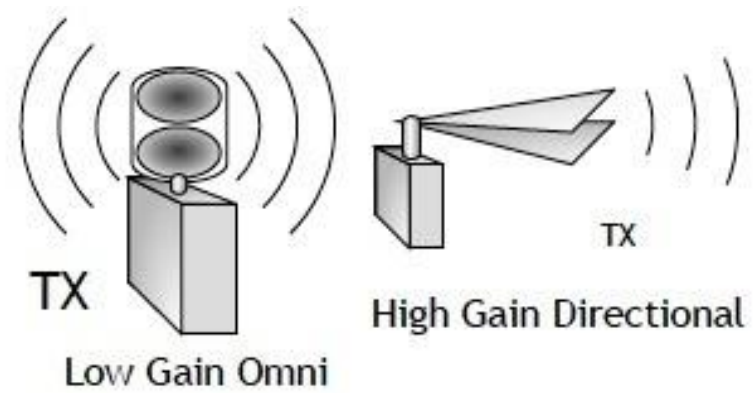

Figure 3: A low gain, omni-directional antenna (left) may be replaced by a high gain directional antenna (right), but transmit power must be reduced to meet the same regulatory limit for peak power. Also, a high gain antenna will tend to be larger and have a narrower field of view than a low gain omni.

\section{2b. Electric or Magnetic Antennas:}

Antennas may also be classified as either electric or magnetic. Electric antennas include dipoles and most horns. These antennas are characterized by intense electric fields close to the antenna. Magnetic antennas include loops and slots. These antennas are characterized by intense magnetic fields close to the antenna.

Electric antennas are more prone to couple to nearby objects than magnetic antennas. Thus, magnetic antennas are preferred for applications involving embedded antennas.

\section{2c. Types of Antennas:}

Many specific kinds of UWB antennas fall within these general categories. Directional antennas include horn and reflector antennas. These antennas can also be implemented in relatively compact planar designs. Small element antennas such as dipoles or loops are preferred for omni-directional coverage or where space is at a particular premium. Traditional "frequency independent" antennas like log periodics or spiral antennas tend to be larger in size and can be used only if waveform dispersion across the field of view may be tolerated. UWB antennas may also be combined in arrays.

System \& Network Considerations for UWB Antennas: Traditional narrowband concepts and techniques often require revision in order to be applied in the UWB context. This section will discuss first the problem of antenna matching, and second, the relationship between directivity and system performance.

3a. Matching \& Spectral Control in UWB Systems: 
Traditionally, a narrowband antenna is treated as a black box with given fixed properties. A system designer either accepts the penalties imposed by antenna shortcomings, or designs a matching network to bridge any impedance gap between the RF front end and the antenna.

A clever matching network can conceal a wealth of antenna sins in the narrowband context. Such matching networks become increasingly difficult to construct as the bandwidth increases. One professor of the author's acquaintance is fond of saying:

\section{"To match to an ultra-wideband antenna one must first start with a well-matched antenna."}

In the UWB context, a good impedance match to an antenna is something that must be designed in from first principles, not added as an afterthought.

The concept of specifically designing an antenna to have a particular impedance has been understood for some time. For instance, Nester disclosed a planar horn antenna with continuously variable elements. This antenna transitions smoothly from a microstrip to a slotline architecture while maintaining an impedance match. Nester's antenna is shown in Figure 4 below.

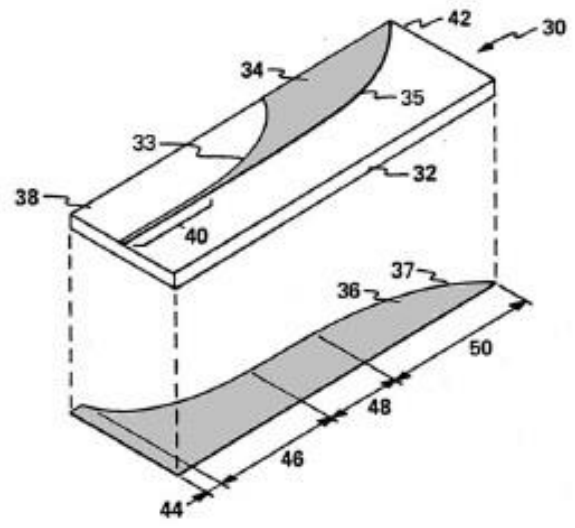

Figure 4: The continuously tapered slot horn elements of Nester (gray colorization on elements added).

Calculating the impedance of a slot line horn requires some complicated algorithms. For simplicity in discussion, assume a parallel plate horn antenna with a cross-sectional width (w) and a height (h). Then the impedance of an air gap horn is approximately given by:

$$
\mathrm{Z}=\mathrm{Z}_{0} \frac{h}{w}
$$

Note that this result is only exact for $\mathrm{w}>>10 \mathrm{~h}$. Since the free space impedance is $\mathrm{Z} 0=377 \Omega, \quad$ a $50 \Omega$ match requires $\mathrm{h} / \mathrm{w} \sim 7.54$ while a $377 \Omega$ match requires $\mathrm{h} / \mathrm{w} \sim 1.00$.

Consider a hypothetical horn antenna matched to $50 \Omega$ at its feed, with a linear transition from $50 \Omega$ to $377 \Omega$, and a long $377 \Omega$ section tapered to be responsive to an ultra-wideband of frequencies. This antenna is shown in Figure 5.

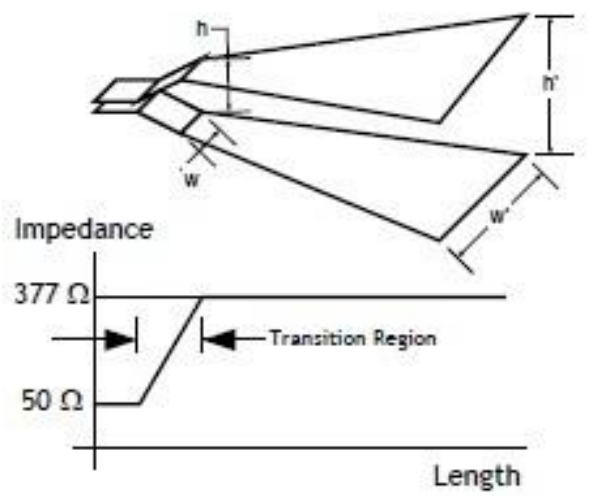

Figure 5: A hypothetical tapered horn antenna (top) with a transition from $50 \Omega$ to $377 \Omega$ (bottom).

Just as a desired impedance can be designed into an antenna, so also can a desired frequency range. The simplest example of this kind of manipulation is to vary the scale of an antenna. For instance, planar elliptical dipoles offer a S11 on the order of $-20 \mathrm{~dB}$ across a 3:1 frequency range. The minor axis is approximately $0.14 \lambda$ at the lower end of the operating band. Thus, a 1-3 GHz antenna will have approximately 1.67 inch elements, a 2-6 GHz antenna will be half the size (one fourth the area) with about 0.83 inch elements, and a $3-9 \mathrm{GHz}$ antenna will be one third the size (one ninth the area) with approximately 0.56 inch elements. The antenna size may be scaled to select any particular 3:1 range of desired frequencies. These antennas are shown in Figure 6.

But this is really just the first step in specifically tailoring an antenna's spectral response to fit a particular design goal. Frequency notches may be implemented using more sophisticated techniques, thus making an antenna insensitive to particular frequencies. Also, the rate of spectral roll-off at the edges of an antenna's operational band may be controlled to some extent.

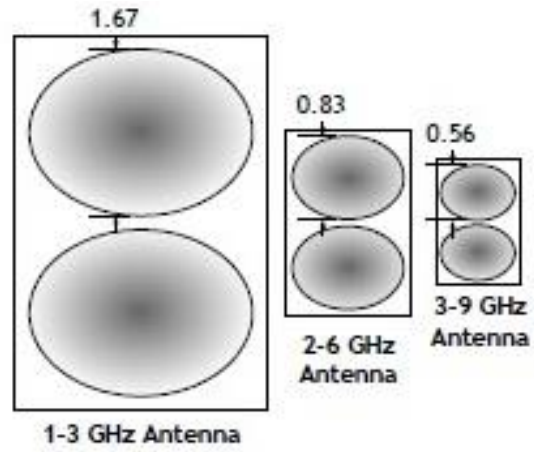

Figure 6: A family of planar elliptical dipole antennas responsive to various frequency ranges. Element size is shown in inches.

While traditional narrowband system design can afford to take a laissez-faire attitude toward antennas, pounding a 
square peg antenna into a round hole RF front end using a matching network hammer, an ultra-wideband system design requires a more holistic approach. A UWB antenna must be specifically tailored in both impedance and spectral response to contribute to the overall system performance.

The concept of using an antenna as a spectral filter is not entirely novel. In spark gap days, RF engineers excited resonant antennas with low frequency broadband impulses, counting on the antenna response to select and radiate the correct frequency components. As we revisit and build upon their pioneering work, we must similarly take advantage of antenna properties to meet system goals.

\section{3b. Directivity and System Performance:}

As with narrowband antennas, the link behavior of UWB antennas in free space is governed by Friis's Law:

where PRX is the received power, PTX is the transmitted power, GTX is the transmit antenna gain, GRX is the receive antenna gain, $\lambda$ is the wavelength, $f$ is the frequency, $\mathrm{c}$ is the speed of light, and $r$ is the range between the antennas. Friis's Law depends on frequency, since in general, power and gain will be functions of frequency. Thus, in the ultra-wideband case, Friis's Law must be interpreted in terms of spectral power density:

$$
d P_{R X}(f)=\frac{c^{2}}{4 \pi r^{2}} P_{T X}(f) \frac{G_{I X}(f) G_{R X}(f)}{f^{2}}
$$

One must integrate over frequency to find the total received power:

$$
P_{R X}=\int_{0}^{\infty} d P_{R X}(f) d f
$$

and the effective isotropic radiated power (EIRP) is:

$$
\operatorname{EIRP}(f)=P_{T X}(f) \cdot G_{T X}(f)
$$

where GTX(f) must be the peak gain of the antenna in any orientation. Since regulatory limits are defined in terms of EIRP, a system designer aims for the product PTX(f) GTX(f) to be constant and as close to the regulatory limit as a reasonable margin of safety (typically $3 \mathrm{~dB}$ ) will allow. Similarly, this power gain product must roll-off so as to fall within the skirts of the allowed spectral mask. Thus, both the antenna designer and transmitter designer must work together to achieve a desired PTX(f) GTX(f), and shortcomings in one spectral response can be made up for and compensated in the other.

Note the dependence of the received power on the inverse frequency squared. Colloquially, this $(\lambda / 4 \pi \mathrm{r}) 2$ or $(\mathrm{c} /(4 \pi \mathrm{rf})) 2$ variation of the signal power is referred to as "path loss." This makes sense (in a way) since the greater the range $r$, the larger the $4 \pi \mathrm{r} 2$ surface area over which a signal is spread and thus the weaker the captured signal. This is more a diffusion of the signal energy than a "loss." Further confusion enters in considering the frequency dependence of "path loss." Interpreting this $1 / \mathrm{f} 2$ dependence as a part of "path loss" suggests that somehow free space attenuates signals in a manner inversely proportional to the square of the frequency. Of course, this is not the case.

The 1/f 2 dependence enters because of the definition of antenna gain and antenna aperture. Antenna gain $\mathrm{G}$ is defined in terms of antenna aperture A as:

$$
G(f)=\frac{4 \pi A(f)}{\lambda^{2}}=\frac{4 \pi A(f) f^{2}}{c^{2}}
$$

This antenna aperture is the effective area of the antenna: a measure of how big a piece of an incoming wave front an antenna can intercept. For directive, electrically large antennas, antenna aperture tends to be comparable to the physical area. For omni-directional small element antennas, the antenna aperture may actually be significantly larger than the antenna's physical area. This follows from the ability of electromagnetic waves to couple to objects within about $\lambda / 2 \pi$. Thus, even though a thin wire or planar antenna may have negligible cross-sectional area, it can still be an effective receiver or radiator of electromagnetic radiation.

The aperture of a constant gain antenna remains constant in units of wavelength. For instance, a dipole antenna has an aperture of approximately $0.132 \lambda 2$. As frequency f increases, $\lambda$ decreases, and the constant gain antenna aperture rolls off as $1 / \mathrm{f} 2$. Typically an omni-directional antenna is designed so as to have constant gain and pattern, and thus, an omnidirectional antenna exhibits this behavior.

Conversely, a "constant aperture" antenna is one whose antenna aperture remains fixed with frequency. For instance, a horn antenna will typically (but not always) have a fixed aperture. As frequency f increases, the size of this aperture in units of wavelength increases as $\mathrm{f} 2$. This narrows the pattern and increases the antenna gain as f 2. Many (but not all) directive antennas exhibit this behavior. Figure 7 shows the pattern behavior of omni vs. directional antennas.

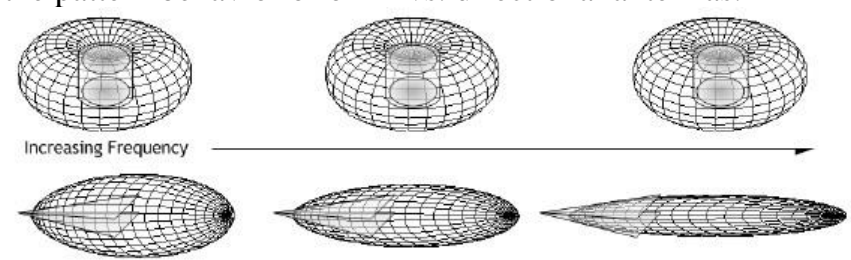

Figure 7: The pattern of a constant gain antenna remains fixed with increasing frequency (top), while the pattern of a constant aperture antenna narrows and gain increases with increasing frequency.

In an omni to omni link, the constant gain antennas on both sides of the link result in the received power rolling off as $1 / \mathrm{f}$ 2 in band. A constant aperture receive antenna whose gain varies as f 2 cancels out this 1/f 2 roll-off and yields a flat received power in band. This received power may be significantly greater than that of a comparable omni antenna depending upon the magnitude of the receive antenna gain. This advantage is offset by a narrowing of the pattern and 
field-of-view that accompanies the increasing gain of a typical directional antenna. Using a directional antenna whose gain varies as f 2 on the transmit side of the link does not improve matters further, because the transmit power must be made to roll-off as 1/f 2 to meet the same flat EIRP spectral mask. Figure 8 depicts this behavior. still seeks to have coded, multi-band or hopping signals yield an average effective power spectrum comparable to that of a broadband impulse implementation.

An FDTD analysis helps in understanding the detailed physics of how signals evolve from a transmit signal via a

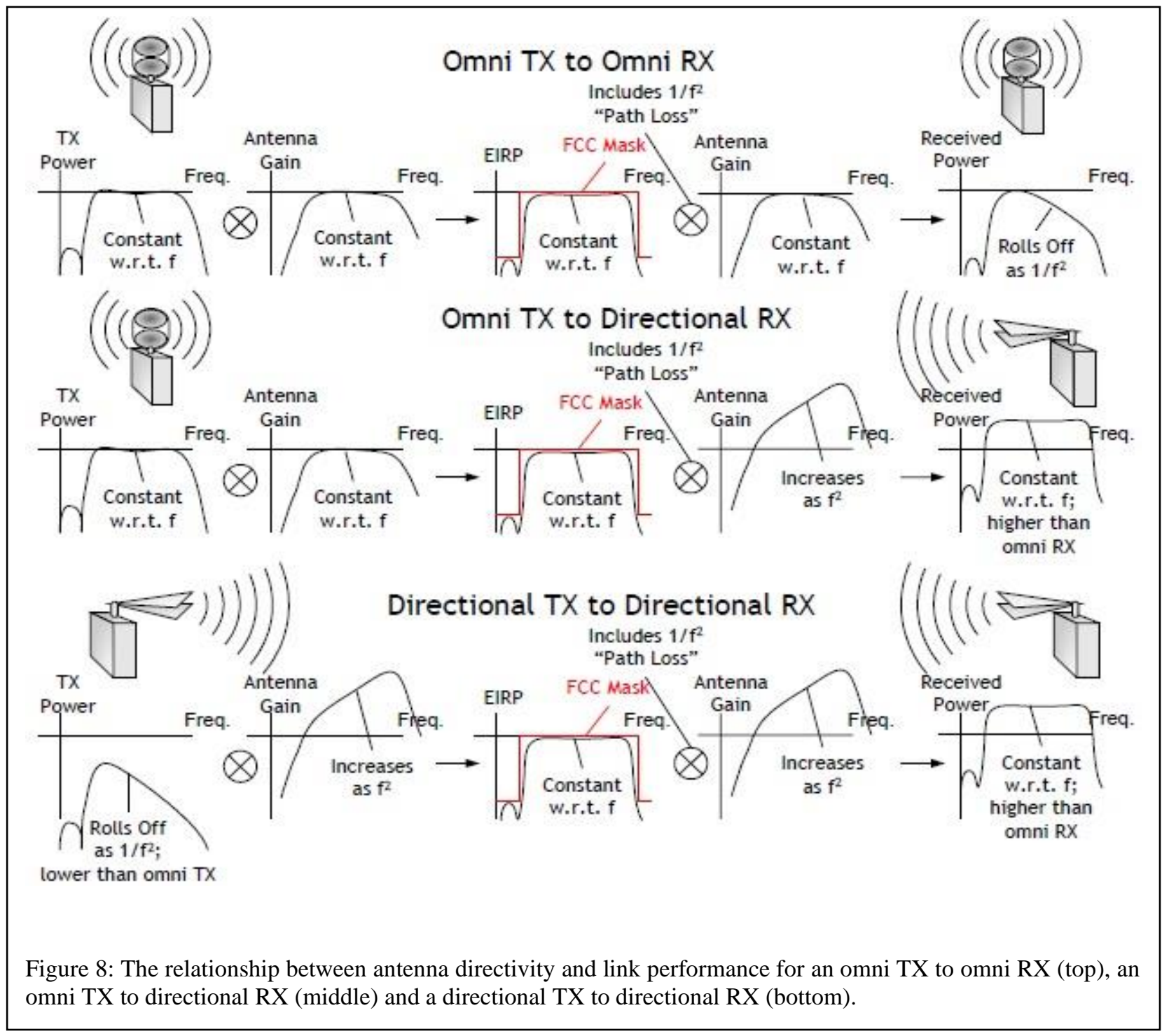

A final potential advantage of directive antennas relative to omni-directional antennas is their ability to isolate signals arriving in particular directions. This ability can be useful in determining the angle of arrival of signals, in applying spatial processing techniques to incoming multi-path signal components, and in nulling out undesired interfering signals. This discussion implicitly assumed a single broadband signal occupying the entire bandwidth. However the general conclusions remain valid for a multi-band or OFDM type implementation. In a multi-band implementation, a designer radiated signal to a received signal. A more detailed physical analysis of radiated and received signals from a typical UWB antenna is available elsewhere.

\section{Fundamental Limits to UWB Antennas:}

This section first considers limits to size and bandwidth of small element dipole antennas. Then, this section presents general guidelines for estimating the gain possible from a particular antenna aperture size.

4a. Antenna Size and Bandwidth: 
Chu explored fundamental limits on antenna size, bandwidth, and efficiency. As generalized by Harrington, Chu's ideas became formalized in the "Chu-Harrington Limit". This limit relates the quality factor " $Q$ " or inverse fractional bandwidth of an ideal, perfectly efficient antenna to its size. Size is denoted by the radius " $r$ " of the boundary sphere: the smallest sphere that completely encloses the antenna (see Figure 9).

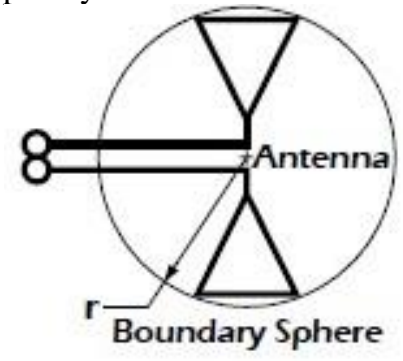

Figure 9: The boundary sphere around an antenna.

The Chu-Harrington limit is:

$$
Q=\frac{1+3(k r)^{2}}{(k r)^{3}\left(1+(k r)^{2}\right)}=\frac{1+3\left(2 \pi r_{\lambda C}\right)^{2}}{\left(2 \pi r_{\lambda C}\right)^{3}\left(1+\left(2 \pi r_{\lambda C}\right)^{2}\right)}=\frac{f_{c}}{\Delta f}
$$

where $\mathrm{k}=2 \pi / \lambda$ is the wave number. The limit may be readily understood when expressed as a function of boundary sphere radius in units of wavelengths at the center frequency, "r $\lambda \mathrm{C}$." The quality factor, "Q," is also defined as the inverse fractional bandwidth, or the ratio of center frequency fc to bandwidth $\Delta \mathrm{f}$.

Recently, the Chu-Harrington limit has been called into question by McLean. McLean points out an error in the derivation of the Chu-Harrington limit and presents a corrected limit:

$$
Q=\frac{1+2(k r)^{2}}{(k r)^{3}\left(1+(k r)^{2}\right)}=\frac{1+2\left(2 \pi r_{i c}\right)^{2}}{\left(2 \pi r_{\lambda C}\right)^{3}\left(1+\left(2 \pi r_{\lambda C}\right)^{2}\right)} \cong \frac{f_{c}}{\Delta f}
$$

For a narrowband antenna there little difference exists between the wavelength $(\lambda \mathrm{C})$ at the center frequency and anywhere else in the operating band.

As the antenna becomes more broadband however, a significant difference emerges between the wavelength $(\lambda \mathrm{H})$ at the high frequency ( $\mathrm{fH}$ ) end of the band, and the wavelength $(\lambda \mathrm{L})$ at the low frequency (fL) end of the band. This difference in wavelength from one end of the band to the other merits examination.

Assume for the sake of argument that the "Q" concept may be extrapolated to the ultra-wideband limit:

Note that in the UWB limit, the center frequency is properly defined as the geometric average Then, the wavelength at either end of an antenna's operating band may be related to the wavelength at the center of the band and the Q. At the high frequency end:

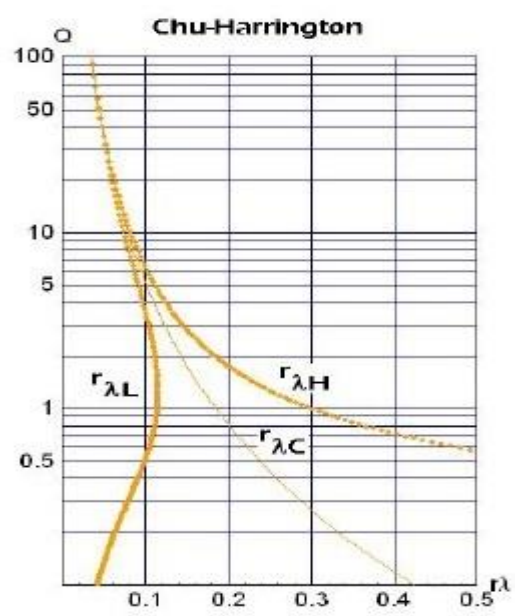

Figure 10: The Chu-Harrington limit. Note the non-physical behavior of r $\lambda \mathrm{L}$.

$$
Q \cong \frac{f_{C}}{\Delta f}=\frac{\sqrt{f_{L} f_{H}}}{f_{H}-f_{L}}
$$

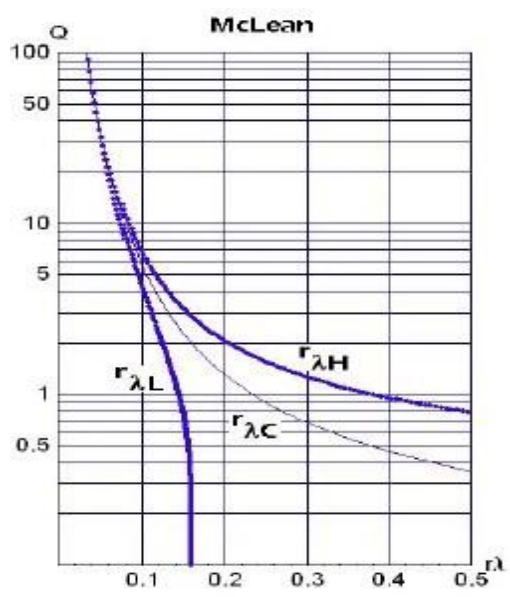

Figure 11: McLean's limit. Note how r $\lambda$ L converges in a wellbehaved manner to the "radiansphere:"

$$
\lambda_{H}=\frac{c}{f_{H}}=\frac{c}{f_{C}} \frac{\sqrt{1+4 Q^{2}}-1}{2 Q}=\lambda_{C} \frac{\sqrt{1+4 Q^{2}}-1}{2 Q}
$$

and at the low frequency end:

$$
\lambda_{L}=\frac{c}{f_{L}}=\frac{c}{f_{C}} \frac{\sqrt{1+4 Q^{2}}+1}{2 Q}=\lambda_{C} \frac{\sqrt{1+4 Q^{2}}+1}{2 Q}
$$

Thus, the characteristic size of an antenna's boundary sphere may be expressed in terms of the wavelength at the center frequency $(\mathrm{r} \lambda \mathrm{C}=\mathrm{r} / \lambda \mathrm{C})$. The wavelength at the high or upper end of the operating band is:

$$
r_{\lambda H}=\frac{r}{\lambda_{H}}=\frac{r}{\lambda_{C}}\left(\frac{2 Q}{\sqrt{1+4 Q^{2}}-1}\right)=r_{\lambda c} \frac{2 Q}{\sqrt{1+4 Q^{2}}-1}
$$

and the wavelength at the lower end of the operating band is: 


$$
r_{\lambda .}=\frac{r}{\lambda_{L}}=\frac{r}{\lambda_{c}}\left(\frac{2 Q}{\sqrt{1+4 Q^{2}}+1}\right)=r_{\lambda c} \frac{2 Q}{\sqrt{1+4 Q^{2}}+1}
$$

The $-3 \mathrm{~dB}$ points relative to the characteristic gain of a constant gain antenna define the start and end of the band. Alternatively, the impedance bandwidth defined by the $-3 \mathrm{~dB}$ S11 points may be used. Defining the bandwidth for a small element antenna can be tricky. Both the Chu-Harrington and McLean analysis start from the assumption of dipole mode behavior. A typical well designed small element dipole antenna will exhibit dipole mode behavior for a 3:1 or more frequency span. At increasingly high frequencies however, this small element antenna will progress out of its dipole mode and into quadropole or higher order modes. Including these higher order modes in determinations of bandwidth may lead to misleading results.

Comparing the Chu-Harrington limit (Figure 10) to the McLean limit (Figure 11) shows a glaring difficulty with the former. The Chu-Harrington limit predicts that antenna size at the low frequency limit makes an unphysical swerve below $\mathrm{Q}=1$. McLean's limit on the other hand converges asymptotically to $\mathrm{r} \lambda \mathrm{C}=1 /(2 \pi)$ in the UWB limit $(\mathrm{Q} \rightarrow 0)$. Interestingly, this corresponds to Wheeler's "radiansphere" radius: $r=\lambda /(2 \pi)$. This is the radial distance at which the reactive and radiative fields are equal in magnitude. The radiansphere defines the boundary between the near or reactive zone and the far or radiation zone about a small antenna.

McLean's limit allows one to establish reasonable expectations on antenna performance. UWB antenna elements preferably span a quarter wavelength or so in dimension at their center frequency. Miniaturizing antennas further requires significant sacrifices in efficiency and performance.

\section{4b. Antenna Size and Gain:}

Equation 6 defines the relation between gain and antenna aperture. As noted earlier, electromagnetic energy readily couples across the "radiansphere" range of $\lambda /(2 \pi)$. Combining these two ideas allows one to establish an approximate bound on the gain possible from an antenna of a particular physical cross-sectional area. The maximum possible antenna aperture approximately equals the physical aperture plus an additional $\lambda /(2 \pi)$ strip around the periphery of the antenna.

Assume an antenna with a circular aperture of physical radius $r$. Then a circular disk of radius $\mathrm{R}=\mathrm{r}+\lambda /(2 \pi)$ bounds the antenna aperture (see Figure 12).

Under these assumptions, Equation 6 allows one to establish an upper bound to antenna gain as a function of physical radius. This relationship is plotted in Figure 13. Similar relationships can be established for other aperture geometries.

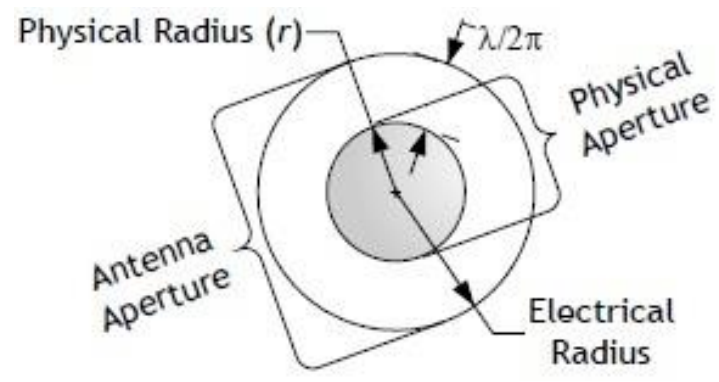

Figure 12: Physical aperture and antenna aperture.

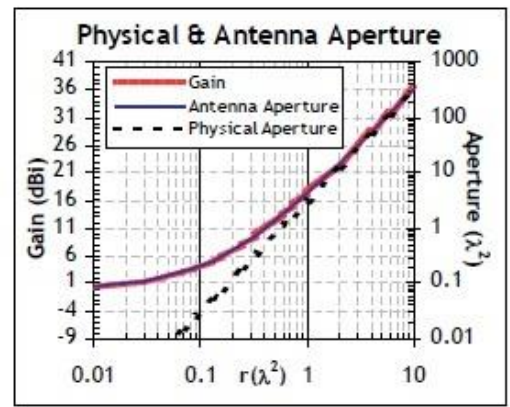

Figure 13: Physical aperture and antenna aperture as a function of physical radius. Note that the primary and second axes have been scaled so as to superimpose the gain and aperture curves.

\section{Conclusion:}

The art and science of UWB antenna design owes much to the pioneers of more traditional antennas. Many of the traditional tradeoffs such as gain vs. field-of-view or antenna size vs. efficiency still apply although influenced by peculiarities of UWB operation such as regulatory constraints.

In some ways, however, UWB antenna design differs profoundly from traditional narrowband design. The spectral and impedance matching properties of a UWB antenna exert a substantial influence on an overall UWB system design. Thus, UWB practice requires a holistic approach to system and antenna design.

Finally, the fundamental constraints imposed by McLean's limit and by the definition of antenna aperture must be considered in setting reasonable expectations for antenna performance.

\section{References:}

[1] K C Gupta et al., Microstrip lines and slotlines, second edition,pp.282-286.

[2] H.Schantz, "Planar Elliptical Element UWB and Dipole Antennas,IEEE APS 2002.

[3] J Foerester et al., "UWB Channel Model Contribution from Intel,” IEEE P802.15-02/279r0-SG3a, June 24,2002.

[4] K Siwiak et al, "FDTD Simulations of UWB Antennas. [5] Hans Gregory Schantz Next-RF, Inc.4811Cove Creek Drive, Brownsboro, AL 35741. 